\title{
On Christol's conjecture
}

\author{
Y. Abdelaziz ${ }^{\dagger}$, C. Koutschanף, J-M. Maillard ${ }^{\dagger}$ \\ $\dagger$ LPTMC, UMR 7600 CNRS, Université Pierre et Marie Curie, Sorbonne \\ Université, Tour 23, 5ème étage, case 121, 4 Place Jussieu, 75252 Paris Cedex \\ 05, France \\ I Johann Radon Institute for Computational and Applied Mathematics, \\ RICAM, Altenberger Strasse 69, A-4040 Linz, Austria
}

\begin{abstract}
We show that the unresolved examples of Christol's conjecture ${ }_{3} F_{2}([2 / 9,5 / 9,8 / 9],[2 / 3,1], x)$ and ${ }_{3} F_{2}([1 / 9,4 / 9,7 / 9],[1 / 3,1], x)$, are indeed diagonals of rational functions. We also show that other ${ }_{3} F_{2}$ and ${ }_{4} F_{3}$ unresolved examples of Christol's conjecture are diagonals of rational functions. Finally we give two arguments that show that it is likely that the ${ }_{3} F_{2}([1 / 9,4 / 9,5 / 9],[1 / 3,1], 27 \cdot x)$ function is a diagonal of a rational function.
\end{abstract}

AMS Classification scheme numbers: 33C05, 33C20, 33F10, 68W30

Key-words: Christol's conjecture, diagonals of rational functions, Shimura curves, creative telescoping, telescopers, D-finite series, globally bounded series.

\section{Introduction}

There is a plethora of multiple integrals in physics: Feynman integrals, lattice Green functions, the summands of the magnetic susceptibility of the 2D Ising model [2, 22, that have very specific mathematical properties. These functions are D-finite, i.e., solutions of linear differential operators with polynomial coefficients, and have series expansions with integer coefficients. Furthermore, it was also shown that the linear differential operators annihilating the summands of the magnetic susceptibility of the Ising model $\tilde{\chi}(n)$, verify the specific property of being Fuchsiant operators: the critical exponents of all their singularities are given by rational numbers, and their Wronskians are $N$-th roots of rational functions [2, 22. It was also shown that the $\tilde{\chi}(n)$ functions are solutions of globally nilpotent operators [5], and that they "come from geometry" being G-operators [25].

The unifying scheme behind these seemingly sparse properties is that these functions are diagonals of rational functions [3, 4. It was shown for example in 4, that if summands of the magnetic susceptibility $\tilde{\chi}(n)$ for any $n$ have an integer coefficient series expansion reducing to algebraic series modulo any prime, it is because they are diagonals of rational functions for any integer $n$. In fact many problems

$\dagger$ Denoting by $\theta$ the homogeneous derivative $x \cdot \frac{d}{d x}$, the degrees of all the polynomial terms of the Fuchsian linear differential operator $\sum_{n} P_{n}(x) \cdot \theta^{n}$ are equal. 
in mathematical physics involving $n$-fold integrals, could be interpreted in terms of diagonals of algebraic or rationa functionst.

Gilles Christol has shown in [9] that for every rational function, its diagonal $f(x)$ has the following properties:

- it is globally bounded: there exist integers $c$ and $d$ in $\mathbb{N}^{*}$, such that $d f(c x) \in$ $\mathbb{Z}[[x]]$ and $f(x)$ has a radius of convergence that is non-zero in $\mathbb{C}$.

- D-finite: there exists a differential operator $L \in \mathbb{Z}[x]\left[\frac{d}{d x}\right]$, with $L \neq 0$, such that $L(f)=0$.

Christol conjectured in [10] that every series that verifies these two properties is the diagonal of a rational function. In this paper [10] Christol came up with an unresolved example to his conjecture [10, and a longer list was generated by Christol and his co-authors in 2012 in [4. In this paper we show that two of the unresolved examples of the conjecture given in [4] on page 58 , namely the ${ }_{3} F_{2}\left([2 / 9,5 / 9,8 / 9],[2 / 3,1], 3^{6} \cdot x\right)$ and ${ }_{3} F_{2}\left([1 / 9,4 / 9,7 / 9],[1 / 3,1], 3^{6} \cdot x\right)$ are indeed diagonals of rational functions and provide a generalization of this result.

\section{Recalls on diagonals of rational functions and on Christol's conjecture}

\subsection{Definition of the diagonal of a rational function}

The diagonal of a rational function in $n$ variables $\mathcal{R}\left(x_{1}, \ldots, x_{n}\right)=$ $\mathcal{P}\left(x_{1}, \ldots, x_{n}\right) / \mathcal{Q}\left(x_{1}, \ldots, x_{n}\right)$, where $\mathcal{P}, \mathcal{Q} \in \mathbb{Q}\left[x_{1}, \ldots, x_{n}\right]$ such that $\mathcal{Q}(0, \ldots, 0) \neq 0$, is defined through its multi-Taylor expansion around $(0, \ldots, 0)$ :

$$
\mathcal{R}\left(x_{1}, \ldots, x_{n}\right)=\sum_{m_{1}=0}^{\infty} \ldots \sum_{m_{n}=0}^{\infty} R_{m_{1}, \ldots, m_{n}} \cdot x_{1}^{m_{1}} \ldots x_{n}^{m_{n}},
$$

as the series in one variable $x$ :

$$
\operatorname{Diag}\left(\mathcal{R}\left(x_{1}, \ldots, x_{n}\right)\right)=\sum_{m=0}^{\infty} R_{m, m, \ldots, m} \cdot x^{m} .
$$

\subsection{Hadamard product of algebraic functions and Christol's conjecture}

Recall that the Hadamard product of two series $f(x)=\sum_{n=0}^{\infty} \alpha_{n} \cdot x^{n}$ and $g(x)=$ $\sum_{n=0}^{\infty} \beta_{n} \cdot x^{n}$ is given by:

$$
f(x) \star g(x)=\sum_{n=0}^{\infty} \alpha_{n} \cdot \beta_{n} \cdot x^{n} .
$$

Hypergeometric series of the form ${ }_{p} F_{p-1}\left(\left[a_{1}, \ldots, a_{p}\right],\left[b_{1} \ldots, b_{p-1}\right], x\right)$ of height $h=h\left(a_{1}, \ldots, a_{p}, b_{1} \ldots, b_{p-1}\right)$, where the height $h$ is given by:

$$
h=\#\left\{1 \leq j \leq p \mid b_{j} \in \mathbb{Z}\right\}-\#\left\{1 \leq j \leq p \mid a_{j} \in \mathbb{Z}\right\}
$$

with $b_{p}=1$, can be written $t$ as the Hadamard product of $h$ globally bounded $\#$ series of height 1, were shown to verify Christol's conjecture. For example, the globally

I Any diagonal of an algebraic function in $n$ variables can be rewritten as the diagonal of a rational function in $2 n$ variables: see [17.

$\dagger$ See 3 4] p.26 and p.58.

$\ddagger$ See 10 p.15.

$\sharp$ Globally bounded series can be recast into series with integer coefficients [3] 4 . 
bounded hypergeometric series ${ }_{3} F_{2}([1 / 3,1 / 3,1 / 3],[1,1], x)$ has height 3 , and it can be written as the Hadamard product of three algebraic functionst:

$$
{ }_{3} F_{2}\left(\left[\frac{1}{3}, \frac{1}{3}, \frac{1}{3}\right],[1,1], x\right)=(1-x)^{-1 / 3} \star(1-x)^{-1 / 3} \star(1-x)^{-1 / 3},
$$

and can thus be written as the diagonal of the algebraic function in three variables:

$$
(1-x)^{-1 / 3} \cdot(1-y)^{-1 / 3} \cdot(1-z)^{-1 / 3} \text {. }
$$

Unlike the case of ${ }_{3} F_{2}([1 / 3,1 / 3,1 / 3],[1,1], x)$, the hypergeometric functions ${ }_{3} F_{2}([2 / 9,5 / 9,8 / 9],[2 / 3,1], x)$ and ${ }_{3} F_{2}([1 / 9,4 / 9,7 / 9],[1 / 3,1], x)$, while being globally bounded functions 12 , were constructed in a way that avoids them being written as "simple" Hadamard products of algebraic functions.

Note that a ${ }_{p} F_{p-1}$ hypergeometric function is globally bounded without restrictions if all its parameters in the denominator are integers, while a ${ }_{p} F_{p-1}$ hypergeometric function can be shown to be globally bounded in general, by looking at Landau functions as explained in the work of Christol [10]. Furthermore, Beukers and Heckman have shown in [7, that ${ }_{p} F_{p-1}$ hypergeometric functions of height zero that are globally bounded are algebraic functions.

\subsection{Unresolved examples to the conjecture}

Generalized hypergeometric functions with regular singularities ${ }_{p} F_{p-1}$ are a simple and natural testing ground for Christol's conjecture.

All ${ }_{2} F_{1}([a, b],[c], x)$ hypergeometric series that are globally bounded are diagonals of rational functions. There are two cases that fall into this category:

- Either the parameter $c$ is an integer, and the ${ }_{2} F_{1}$ function can be written as the Hadamard product of two ${ }_{1} F_{0}$ functions, which are algebraic functions, and thus are diagonals of rational functions by Furstenberg's [15] theoren 4 .

- The parameter $c$ is not an integer, and in this case the ${ }_{2} F_{1}$ function is a diagonal of a rational function if and only if it is an algebraic function tit (and consequently its series is globally bounded).

Moving on to ${ }_{3} F_{2}$ hypergeometric functions, one can ask when is a ${ }_{3} F_{2}$ hypergeometric function a diagonal of a rational function?

- When the parameters $d$ and $e$ in ${ }_{3} F_{2}([a, b, c],[d, e], x)$ are integers, because, in this case, the ${ }_{3} F_{2}$ can be written as the Hadamard product of three ${ }_{1} F_{0}$ algebraic functions, and is thus the diagonal of a rational function, by the closure of diagonals under the Hadamard product.

- When the parameters $d$ and $e$ in ${ }_{3} F_{2}([a, b, c],[d, e], x)$ are rational numbers but not integers, because in this case the ${ }_{3} F_{2}$ is algebraic, and is thus a diagonal by Furstenberg's theorem.

$\dagger$ Diagonals are closed under the Hadamard product: if two series are diagonals of rational functions, their Hadamard product is also a diagonal of a rational function.

I Furstenberg's theorem states that any algebraic function is the diagonal of a rational function in two variables.

††The only ${ }_{2} F_{1}$ hypergeometric functions that are globally bounded with $c \in \mathbb{Q}$ are the algebraic ones: they are the ones appearing in the list of Schwarz 13 . 
Hence the interesting case occurs when only one of the two parameters $d$ or $e$ is rational, and the other is an integer. But even in this case, a lot of the ${ }_{3} F_{2}$ functions are easily seen to be diagonals of a rational function. Suppose that a ${ }_{3} F_{2}([a, b, c],[1, e], x)$ is globally bounded, with the parameters $a, b, c, e \in \mathbb{Q} \backslash \mathbb{Z}$, then there are six ways to write the ${ }_{3} F_{2}([a, b, c],[1, e], x)$ function as the diagonal of a rational function. This corresponds to the six ways to write the ${ }_{3} F_{2}([a, b, c],[1, e], x)$ as a Hadamard product of hypergeometric functions:

- ${ }_{2} F_{1}([a, b],[e], x) \star{ }_{1} F_{0}([c], x)$

- ${ }_{2} F_{1}([a, c],[e], x) \star{ }_{1} F_{0}([b], x)$

- ${ }_{2} F_{1}([b, c],[e], x) \star{ }_{1} F_{0}([a], x)$

- ${ }_{2} F_{1}([a, b],[1], x) \star{ }_{2} F_{1}([c, 1],[e], x)$

- ${ }_{2} F_{1}([a, c],[1], x) \star{ }_{2} F_{1}([b, 1],[e], x)$

- ${ }_{2} F_{1}([b, c],[1], x) \star{ }_{2} F_{1}([a, 1],[e], x)$

Now ${ }_{1} F_{0}([c], x)$ and ${ }_{2} F_{1}([a, b],[1], x)$ are diagonals of rational functions by what we have said above. Then ${ }_{3} F_{2}([a, b, c],[1, e], x)$ is a diagonal of rational functions if ${ }_{2} F_{1}([c, 1],[e], x)$ or ${ }_{2} F_{1}([a, b],[e], x)$ t and only if they are algebraic functions, since $e \in \mathbb{Q} \backslash \mathbb{Z}$. Now ${ }_{2} F_{1}([c, 1],[e], x)$ cannot be an algebraic functions for $e \in \mathbb{Q}$ by Goursat [6]. Hence if one of ${ }_{2} F_{1}([a, b],[e], x),{ }_{2} F_{1}([b, c],[e], x)$, or ${ }_{2} F_{1}([a, c],[e], x)$ is an algebraic function, then ${ }_{3} F_{2}([a, b, c],[1, e], x)$ is the diagonal of a rational function. Now taking the two examples given in [4 or by G. Christol in [11] that we are looking at here, we see that neither ${ }_{3} F_{2}([2 / 9,5 / 9,8 / 9],[2 / 3,1], x)$, nor ${ }_{3} F_{2}([1 / 9,4 / 9,7 / 9],[2 / 3,1], x)$, can be obtained as diagonals of rational functions through Hadamard product $t \rightarrow$ since the three ${ }_{2} F_{1}$ hypergeometric series are not globally bounded:

$$
{ }_{2} F_{1}\left(\left[\frac{2}{9}, \frac{5}{9}\right],\left[\frac{2}{3}\right], x\right), \quad{ }_{2} F_{1}\left(\left[\frac{2}{9}, \frac{8}{9}\right],\left[\frac{2}{3}\right], x\right), \quad{ }_{2} F_{1}\left(\left[\frac{5}{9}, \frac{8}{9}\right],\left[\frac{2}{3}\right], x\right),
$$

and nor are the ${ }_{2} F_{1}$ hypergeometric series:

$$
{ }_{2} F_{1}\left(\left[\frac{1}{9}, \frac{4}{9}\right],\left[\frac{1}{3}\right], x\right), \quad{ }_{2} F_{1}\left(\left[\frac{4}{9}, \frac{7}{9}\right],\left[\frac{1}{3}\right], x\right), \quad{ }_{2} F_{1}\left(\left[\frac{1}{9}, \frac{7}{9}\right],\left[\frac{1}{3}\right], x\right) .
$$

\section{The main results}

The globally bounded ${ }_{3} F_{2}$ hypergeometric series

$$
{ }_{3} F_{2}\left(\left[\frac{2}{9}, \frac{5}{9}, \frac{8}{9}\right],\left[\frac{2}{3}, 1\right], 27 \cdot x\right), \quad{ }_{3} F_{2}\left(\left[\frac{1}{9}, \frac{4}{9}, \frac{7}{9}\right],\left[\frac{1}{3}, 1\right], 27 \cdot x\right)
$$

ar $\$$ respectively the diagonals of the two algebraic functions

$$
{ }_{3} F_{2}\left(\left[\frac{2}{9}, \frac{5}{9}, \frac{8}{9}\right],\left[\frac{2}{3}, 1\right], 27 \cdot x\right)=\operatorname{Diag}\left(\frac{(1-x-y)^{1 / 3}}{1-x-y-z}\right),
$$

$\dagger \dagger$ Instead of ${ }_{2} F_{1}([c, 1],[e], x)$, or one could take any one of the three permuted versions: ${ }_{2} F_{1}([b, 1],[e], x)$, etc.

$\#$ Appendix F p.58 of [4], also see 11 p.19.

$\dagger$ See 23 for a proof that ${ }_{3} F_{2}([1 / 9,4 / 9,7 / 9],[2 / 3,1], x)$ cannot be written as a Hadamard product. I One can see this experimentally by taking the series expansion of any of the Gauss hypergeometric functions: the prime numbers in the denominators of the coefficients grow continuously.

$\ddagger$ The operators annihilating the two hypergeometric functions (9) are adjoint of each other. 
and

$$
{ }_{3} F_{2}\left(\left[\frac{1}{9}, \frac{4}{9}, \frac{7}{9}\right],\left[\frac{1}{3}, 1\right], 27 \cdot x\right)=\operatorname{Diag}\left(\frac{(1-x-y)^{2 / 3}}{1-x-y-z}\right) .
$$

These two hypergeometric serie:t1 (9) can be recast into series with integer coefficients

$$
{ }_{3} F_{2}\left(\left[\frac{2}{9}, \frac{5}{9}, \frac{8}{9}\right],\left[\frac{2}{3}, 1\right], 3^{6} \cdot x\right)=1+120 x+47124 x^{2}+23483460 x^{3}+\cdots,
$$

and

$$
{ }_{3} F_{2}\left(\left[\frac{1}{9}, \frac{4}{9}, \frac{7}{9}\right],\left[\frac{1}{3}, 1\right], 3^{6} \cdot x\right)=1+84 x+32760 x^{2}+16302000 x^{3}+\cdots
$$

Now Denef and Lipshitz in [17] show that any power series in $\mathbb{Q}\left[\left[x_{1}, \ldots, x_{n}\right]\right]$, algebraic over $\mathbb{Q}\left(x_{1}, \ldots, x_{n}\right)$, is the diagonal of a rational function in $2 n$ variables, and they give an algorithm to build this rational function. This means that we can construct the rational functions, whose corresponding diagonals are the ${ }_{3} F_{2}([2 / 9,5 / 9,8 / 9],[2 / 3,1], 27 \cdot x)$ and the ${ }_{3} F_{2}([1 / 9,4 / 9,7 / 9],[1 / 3,1], 27 \cdot x)$ functions. We recall the algorithm of Denef and Lipshitz and apply it to the algebraic function $(1-x-y)^{1 / 3} /(1-x-y-z)$ in the first subsection below, and then we give the rational function and a generalization of the result in the second subsection. Finally, we give a second proof of the general result using binomial sums.

\subsection{From diagonals of algebraic functions to diagonals of rational functions: Denef} and Lipshitz

We explain a method which, for a given algebraic power series in $n$ variables, constructs a rational function in $2 n$ variables whose diagonal equals the diagonal of the given algebraic series. Moreover, the partial diagonal of that $2 n$-variable rational function, with respect to the pairs of variables $\left(x_{1}, x_{n+1}\right), \ldots,\left(x_{n-1}, x_{2 n}\right)$, yields the original $n$-variable algebraic power series. The method is described in the paper by Denef and Lipshitz [17] in the proof of their Theorem 6.2. As a running example we use the three-variable algebraic function

$$
f(x, y, z)=\frac{(1-x-y)^{1 / 3}}{1-x-y-z}
$$

whose multi-Taylor series expansion at 0 is actually a power series in the three variables $x, y, z$ :

$$
f(x, y, z)=1+\frac{2}{3} x+\frac{2}{3} y+z+\frac{10}{9} x y+\frac{5}{3} x z+\frac{5}{3} y z+\frac{40}{9} x y z+\ldots
$$

Note that the minimal polynomial of $f$ is given by

$$
p(x, y, z, f)=((x+y+z-1) \cdot f)^{3}+1-x-y .
$$

Denef and Lipshitz's theorem is formulated for étale extensions, which basically means that the partial derivative (w.r.t. $f$ ) of the minimal polynomial has a nonzero constant coefficient at 0 . Clearly, the above polynomial $p(x, y, z, f)$ does not meet this criterion.

†† The hypergeometric function ${ }_{3} F_{2}([2 / 9,5 / 9,8 / 9],[2 / 3,1], 27 x)$ can be rewritten as the Hadamard product ${ }_{2} F_{1}\left(\left[\frac{2}{9}, \frac{5}{9}\right],\left[\frac{2}{3}\right], 27 x\right) \star(1-x)^{-8 / 9}$ with ${ }_{2} F_{1}\left(\left[\frac{2}{9}, \frac{5}{9}\right],\left[\frac{2}{3}\right], 27 x\right)$ being associated with a Shimura curve [24]. For more details please refer to Appendix A 
However, by considering $\tilde{f}=f-1$, i.e. by removing the constant term of $f$, we can achieve an étale extension. The minimal polynomial then reads

$$
\tilde{p}(x, y, z, f)=((x+y+z-1) \cdot(f+1))^{3}+1-x-y .
$$

Indeed, $\frac{\partial \tilde{p}}{\partial f}(0,0,0,0)=-3 \neq 0$. According to the proof of Theorem 6.2 (i) in [17, the rational function

$$
\tilde{r}(x, y, z, f)=f^{2} \cdot \frac{\frac{\partial \tilde{p}}{\partial f}(x f, y f, z f, f)}{\tilde{p}(x f, y f, z f, f)}
$$

has the property that $\mathcal{D}(\tilde{r}(x, y, z, f))=\tilde{f}(x, y, z)$, and hence $\mathcal{D}(r(x, y, z, f))=$ $f(x, y, z)$ for $r(x, y, z, f)=\tilde{r}(x, y, z, f)+1$. Here the operator $\mathcal{D}$ denotes a special kind of "diagonalization" with respect to the last variable: for

$$
f\left(x_{1}, \ldots, x_{n}, y\right)=\sum a_{i_{1}, \ldots, i_{n}, j} \cdot x_{1}^{i_{1}} \cdots x_{n}^{i_{n}} y^{j},
$$

one defines

$$
\mathcal{D}\left(f\left(x_{1}, \ldots, x_{n}, y\right)\right)=\sum_{j=i_{1}+\cdots+i_{n}} a_{i_{1}, \ldots, i_{n}, j} \cdot x_{1}^{i_{1}} \cdots x_{n}^{i_{n}} .
$$

In our running example we obtain:

$$
r(x, y, z, f)=\frac{3 f^{2} \cdot(f+1)^{2} \cdot(x f+y f+z f-1)^{3}}{(f+1)^{3} \cdot(x f+y f+z f-1)^{3}-x f-y f+1}+1 .
$$

In the second step, which is explained in the proof of Theorem 6.2(ii) of [17], one has to transform the rational function $r$ (that has $n+1$ variables) into another rational function (having $2 n$ variables) such that its "true" (partial) diagonal gives the $n$ variable algebraic series $f$. It consists of a sequence of $n-1$ elementary steps, each of which is adding one more variable. In our example, we have to do the following

$$
\begin{aligned}
& r_{1}\left(x, y, z, u_{1}, v_{1}\right)=\frac{u_{1} \cdot r\left(x, y, z, u_{1}\right)-v_{1} \cdot r\left(x, y, z, v_{1}\right)}{u_{1}-v_{1}}, \\
& r_{2}\left(x, y, z, u_{1}, u_{2}, v_{2}\right)=\frac{u_{2} \cdot r_{1}\left(x, y, z, u_{1}, u_{2}\right)-v_{2} \cdot r_{1}\left(x, y, z, u_{1}, v_{2}\right)}{u_{2}-v_{2}},
\end{aligned}
$$

and obtain with $r_{2}$ the desired rational function in six variables.

\subsection{Generalization of the previous result}

By the algorithm of Denef and Lipshitz given in the previous section, it is possible to show that the algebraic function

$$
\frac{(1-x-y)^{a / b}}{1-x-y-z}
$$

corresponds to the following rational function in six variables, by taking the diagonal with respect to $(x, u),(y, v)$ and $(z, w)$ :

$$
\begin{aligned}
& \frac{a \cdot u^{3} v \cdot(1-u x-u y-u z) \cdot(1+u)^{a-1} \cdot(1-u x-u y-u z)^{a-1}}{(1+u)^{a} \cdot(1-u x-u y-u z)^{a}-(1-u x-u y)^{b} \cdot(u-v) \cdot(v-w)} \\
& -\frac{a \cdot v^{4} \cdot(1-v x-v y-v z) \cdot((1+v) \cdot(1-v x-v y-v z))^{a-1}}{(1+v)^{a} \cdot(1-v x-v y-v z)^{a}-(1-v x-v y)^{b} \cdot(u-v)(v-w)} \\
& -\frac{a \cdot u^{3} w \cdot(1-u x-u y-u z)((1+u) \cdot(1-u x-u y-u z))^{a-1}}{(1+u)^{a} \cdot(1-u x-u y-u z)^{a}-(1-u x-u y)^{b} \cdot(u-w) \cdot(v-w)} \\
& \quad-\frac{a w^{4} \cdot(1-w x-w y-w z) \cdot(1+w)^{a-1} \cdot(1-w x-w y-w z)^{a-1}}{(1+w)^{a} \cdot(1-w x-w y-w z)^{a}-(1-w x-w y)^{b} \cdot(u-w) \cdot(v-w)}+1 .
\end{aligned}
$$


The diagonal of the rational function (24) is annihilated by the linear differential operator of order three:

$$
\begin{aligned}
& b^{3} x^{2}(1-27 x) \cdot D_{x}^{3}+b^{2} x((27 a-135 b) \cdot x-a+3 b) \cdot D_{x}^{2} \\
& \quad-b \cdot\left(\left(9 a^{2}-63 a b+114 b^{2}\right) \cdot x+a b-b^{2}\right) \cdot D_{x}+(a-3 b) \cdot(a-2 b) \cdot(a-b),
\end{aligned}
$$

and can be expressed as the ${ }_{3} F_{2}$ hypergeometric function

$$
{ }_{3} F_{2}\left(\left[\frac{3 a-b}{3 a}, \frac{2 a-b}{3 a}, \frac{a-b}{3 a}\right],\left[\frac{a-b}{a}, 1\right], 27 \cdot x\right) .
$$

In particular, the two hypergeometric functions ${ }_{3} F_{2}([2 / 9,5 / 9,8 / 9],[2 / 3,1], 27 \cdot x)$ and ${ }_{3} F_{2}([1 / 9,4 / 9,7 / 9],[1 / 3,1], 27 \cdot x)$ appearing in (9), correspond respectively to the parameters $(a, b)=(1,3)$, and $(a, b)=(2,3)$ in the algebraic function (23). Other values of the parameters $(a, b)$ are not necessarily unresolved examples of Christol's conjecture.

For example if we consider the parameter values $a=1$ and $b=7$, we see that the diagonal of (24) is given by the globally boundedt) series (27)

${ }_{3} F_{2}\left(\left[\frac{2}{7}, \frac{13}{21}, \frac{20}{21}\right],\left[\frac{6}{7}, 1\right], 27 x\right)=1+\frac{260}{49} x+\frac{188190}{2401} x^{2}+\cdots$

with the ${ }_{2} F_{1}$ series

$$
{ }_{2} F_{1}\left(\left[\frac{13}{21}, \frac{20}{21}\right],\left[\frac{6}{7}\right], 27 x\right), \quad{ }_{2} F_{1}\left(\left[\frac{2}{7}, \frac{20}{21}\right],\left[\frac{6}{7}\right], 27 x\right), \quad{ }_{2} F_{1}\left(\left[\frac{2}{7}, \frac{13}{21}\right],\left[\frac{6}{7}\right], 27 x\right),
$$

being series that are not globally bounded. Hence the hypergeometric series (27) cannot be easily written as a Hadamard product, as explained in Section 2.3.

In contrast, for $a=3$ and $b=4$ the diagonal of (24) which is given by the globally bounded series (28)

$$
{ }_{3} F_{2}\left(\left[\frac{3}{4}, \frac{5}{12}, \frac{1}{12}\right],\left[\frac{1}{4}, 1\right], 27 x\right)=1+\frac{45}{16} x+\frac{41769}{1024} x^{2}+\cdots
$$

with the ${ }_{2} F_{1}$ series

$$
{ }_{2} F_{1}\left(\left[\frac{5}{12}, \frac{1}{12}\right],\left[\frac{1}{4}\right], 27 x\right),
$$

being a globally bounded series, which means that it can be written as a diagonal using one of the procedures given in Section 2.3. We note that algebraic functions close to the algebraic functions appearing in (10) and (11), also give ${ }_{3} F_{2}$ or ${ }_{4} F_{3}$ hypergeometric functions as their diagonals that are unresolved examples to Christol's conjecture:

$$
\begin{aligned}
& \operatorname{Diag}\left(\frac{(1-x-2 y)^{2 / 3}}{1-x-y-z}\right)={ }_{3} F_{2}\left(\left[\frac{1}{9}, \frac{4}{9}, \frac{7}{9}\right],\left[\frac{2}{3}, 1\right], 27 \cdot x\right), \\
& \operatorname{Diag}\left(\frac{(1-x-2 y)^{1 / 3}}{1-x-y-z}\right)={ }_{3} F_{2}\left(\left[\frac{2}{9}, \frac{5}{9}, \frac{8}{9}\right],\left[\frac{5}{6}, 1\right], 27 \cdot x\right), \\
& \operatorname{Diag}\left(\frac{(1-x)^{1 / 3}}{1-x-y-z}\right)={ }_{4} F_{3}\left(\left[\frac{2}{9}, \frac{5}{9}, \frac{8}{9}, \frac{1}{2}\right],\left[\frac{1}{3}, \frac{5}{6}, 1\right], 27 \cdot x\right), \\
& \operatorname{Diag}\left(\frac{(1-x-y)^{1 / 3}}{1-x-z}\right)={ }_{4} F_{3}\left(\left[\frac{2}{9}, \frac{5}{9}, \frac{8}{9}, \frac{-1}{3}\right],\left[\frac{1}{3}, \frac{5}{6}, 1\right], 27 \cdot x\right) .
\end{aligned}
$$

$\dagger_{3} F_{2}\left(\left[\frac{2}{7}, \frac{13}{21}, \frac{20}{21}\right],\left[\frac{6}{7}, 1\right], 27 \cdot 7^{3} \cdot x\right)$ is a series with integer coefficients.

$\ddagger{ }_{3} F_{2}\left(\left[\frac{3}{4}, \frac{5}{12}, \frac{1}{12}\right],\left[\frac{1}{4}, 1\right], 1728 \cdot x\right)$ is a series with integer coefficients. 


\subsection{Proof}

A computer algebra proof of this result can easily be obtained using the creative telescoping program [16]: one computes the operator (25) using the program [16], and verifies that this operator does annihilate the diagonal of (23) tit. Another longer way to do it which we provide below, is through binomial sums.

The denominator of the algebraic function $(1-x-y)^{a / b} /(1-x-y-z)$ can be expanded as a geometric series:

$$
\begin{aligned}
(1-x-y-z)^{-1} & =\sum_{n=0}^{\infty} \sum_{m=0}^{\infty}\left(\begin{array}{c}
n \\
m
\end{array}\right) \cdot(x+y)^{m} z^{n-m} \\
& =\sum_{n=0}^{\infty} \sum_{m=0}^{\infty} \sum_{l=0}^{\infty}\left(\begin{array}{c}
n \\
m
\end{array}\right)\left(\begin{array}{c}
m \\
l
\end{array}\right) \cdot x^{l} y^{m-l} z^{n-m},
\end{aligned}
$$

while the numerator can be written as the sum:

$$
(1-(x+y))^{a / b}=\sum_{k=0}^{\infty} \frac{(-a / b)_{k}}{k !} \cdot(x+y)^{k}=\sum_{k=0}^{\infty} \sum_{j=0}^{k} \frac{(-a / b)_{k}}{k !} \cdot\left(\begin{array}{l}
k \\
j
\end{array}\right) x^{j} y^{k-j} .
$$

Multiplying these two sums (34) and (35) and re-indexing, we obtain:

$$
\sum_{s=0}^{\infty} \sum_{t=0}^{\infty} \sum_{u=0}^{\infty} x^{s} y^{t} z^{u} \cdot \sum_{j=0}^{s} \sum_{k=0}^{\infty} \frac{(-a / b)_{k}}{k !} \cdot\left(\begin{array}{c}
k \\
j
\end{array}\right)\left(\begin{array}{c}
s+t+u-k \\
s+t-k
\end{array}\right)\left(\begin{array}{c}
s+t-k \\
s-j
\end{array}\right) .
$$

Now taking the coefficients corresponding to the diagonal in (36), i.e. such that $s=t=u=n$, we get:

$$
\begin{aligned}
\sum_{j=0}^{n} \sum_{k=0}^{\infty} \frac{(-a / b)_{k}}{k !} \cdot\left(\begin{array}{c}
k \\
j
\end{array}\right)\left(\begin{array}{c}
3 n-k \\
2 n-k
\end{array}\right)\left(\begin{array}{c}
2 n-k \\
n-j
\end{array}\right) \\
=\sum_{k=0}^{2 n} \frac{(-a / b)_{k}}{k !} \cdot\left(\begin{array}{c}
3 n-k \\
2 n-k
\end{array}\right) \cdot \sum_{j=0}^{n}\left(\begin{array}{c}
k \\
j
\end{array}\right)\left(\begin{array}{c}
2 n-k \\
n-j
\end{array}\right) .
\end{aligned}
$$

Now recalling the Chu-Vandermonde identity which says that $\left(\begin{array}{c}2 n \\ n\end{array}\right)=$ $\sum_{j=0}^{n}\left(\begin{array}{c}k \\ j\end{array}\right)\left(\begin{array}{c}2 n-k \\ n-j\end{array}\right)$, we find that (37) can be written as

$$
S(n)=\left(\begin{array}{c}
2 n \\
n
\end{array}\right) \cdot \sum_{k=0}^{2 n} \frac{(-a / b)_{k}}{k !} \cdot\left(\begin{array}{c}
3 n-k \\
2 n-k
\end{array}\right)
$$

and by using a computer algebra tool like Mathematica or Maple to simplify this sum into a closed form, from which we can read off the hypergeometric function representation of the diagonal. More precisely, we used creative telescoping (Zeilberger's algorithm) to prove that (38) satisfies the first-order recurrence:

$$
\begin{gathered}
(a-3 b-3 b n) \cdot(a-2 b-3 b n) \cdot(a-b-3 b n) \cdot S(n) \\
=b^{2} \cdot(n+1)^{2} \cdot(a-b-b n) \cdot S(n+1) .
\end{gathered}
$$

Together with the initial condition $S(0)=1$, we obtain the closed form

$$
S(n)=\frac{3^{3 n} \cdot((b-a) / 3 b)_{n} \cdot((2 b-a) / 3 b)_{n} \cdot((3 b-a) / 3 b)_{n}}{((b-a) / b)_{n} \cdot(n !)^{2}} .
$$

$\dagger \dagger$ One also needs to note that initial conditions have to be compared. 


\section{Telescopers of algebraic functions versus diagonals of algebraic functions}

The diagonal of a rational function and a solution of a telescopent of a rational function are close, yet distinct notions. A telescoper annihilates an $n$-fold integral of a rational function over all integration cyclestit. For example the ${ }_{3} F_{2}([a, b, c],[d, 1], x)$ can be written through the well-known integral representation as:

$$
(1-y)^{-1-b+d} \cdot y^{b} \cdot\left(1-x \cdot y^{2}\right)^{-a} \cdot(1-z)^{-c},
$$

with $a, b, c, d \in \mathbb{Q}$. Hence if one takes the parameters $a, b, c, d$ to have the values $a=1 / 9, b=4 / 9, c=7 / 9, d=1 / 3$, one immediately obtains that the telescoper of the algebraic function

$$
\frac{y^{4 / 9}}{(1-y)^{10 / 9} \cdot\left(1-x y^{2}\right)^{1 / 9} \cdot(1-z)^{7 / 9}},
$$

admits as a solution the hypergeometric function ${ }_{3} F_{2}\left(\left[\frac{1}{9}, \frac{4}{9}, \frac{7}{9}\right],\left[\frac{1}{3}, 1\right], x\right)$. Yet the diagonal of the algebraic function (42) is equal to zero, and it is through the algebraic function (11) that we were able to obtain it as the diagonal of an algebraic function. Other ${ }_{3} F_{2}$ unresolved examples to Christol's conjecture like [10, 11]

$$
{ }_{3} F_{2}\left(\left[\frac{1}{9}, \frac{4}{9}, \frac{5}{9}\right],\left[\frac{1}{3}, 1\right], 27 \cdot x\right)
$$

were not obtained here as diagonals of a rational function, yet they are solution of the telescoper of an algebraic function and can thus be seen as a period of an algebraic variety over a non-evanescent cycl $₫$, but not necessarily as a diagonal of an algebraic function (i.e. a period over an evanescent cycle). We give two arguments in favour of the fact that the ${ }_{3} F_{2}$ hypergeometric function (43) is most probably a diagonal of an algebraic function.

\subsection{Diagonal: algebraic $\bmod p$}

If one expects ${ }_{3} F_{2}$ hypergeometric functions like (43) to be diagonals of an algebraic function, one should find 3 , 4, that the corresponding series expansion reduces to an algebraic series modulo any prime number $p$, or power of a prime number $p^{r}$. In order to verify this fact on (43) we look at the series expansion of

$$
\begin{aligned}
{ }_{3} F_{2}\left(\left[\frac{1}{9}, \frac{4}{9}, \frac{5}{9}\right],\left[\frac{1}{3}, 1\right], 27^{2} \cdot x\right)=1+60 x+20475 x^{2}+9373650 x^{3} \\
+4881796920 x^{4}+2734407111744 x^{5}+1605040007778900 x^{6}+\cdots
\end{aligned}
$$

which becomes modulo 2 :

$$
\begin{aligned}
{ }_{3} F_{2} & \left(\left[\frac{1}{9}, \frac{4}{9}, \frac{5}{9}\right],\left[\frac{1}{3}, 1\right], 27^{2} \cdot x\right)=1+x^{2}+x^{128}+x^{130} \\
& +x^{8192}+x^{8194}+x^{8320}+x^{8322} \\
& +x^{524288}+x^{524290}+x^{524416}+x^{524418}+x^{532480}+x^{532482}+x^{532608}+x^{532610} \\
\quad & \quad+O\left(x^{600000}\right) \\
= & \left(1+x^{2}\right) \cdot\left(1+x^{128}\right) \cdot\left(1+x^{8192}\right) \cdot\left(1+x^{524288}\right) \quad+O\left(x^{600000}\right) .
\end{aligned}
$$

$\dagger$ By "telescoper" of a rational function $R(x, y, z)$ we denote the output of the creative telescoping program [16], applied to the transformed rational function $R(x / y, y / z, z) /(y z)$, which is a differential operator that annihilates the diagonal of $R$.

$\dagger \dagger$ Diagonals correspond only to evanescent integration cycles over rational functions.

$\ddagger$ To be totally rigorous, one has to consider the two certificates of the telescoping equation see if that the integral of the derivatives of these two certificates over that cycle are actually zero. 
Straightforward guessing gives the infinite product formula

$$
F(x)=\left(1+x^{2}\right) \cdot\left(1+x^{2^{7}}\right) \cdot\left(1+x^{2^{13}}\right) \cdot\left(1+x^{2^{19}}\right) \cdots\left(1+x^{2^{6 n+1}}\right) \cdots
$$

which is solution of

$$
F(x)=\left(1+x^{2}\right) \cdot F\left(x^{64}\right) \quad \bmod . \quad 2,
$$

i.e. ${ }_{3} F_{2}\left(\left[\frac{1}{9}, \frac{4}{9}, \frac{5}{9}\right],\left[\frac{1}{3}, 1\right], 27^{2} \cdot x\right)$ is an algebraic function modulo 2 satisfying:

$$
F(x)=\left(1+x^{2}\right) \cdot F(x)^{64} \quad \bmod .2
$$

or:

$$
\left(1+x^{2}\right) \cdot F(x)^{63}=1 \quad \bmod .2 .
$$

Modulo 3 we have the following expansion

$$
\frac{{ }_{3} F_{2}\left(\left[\frac{1}{9}, \frac{4}{9}, \frac{5}{9}\right],\left[\frac{1}{3}, 1\right], 27^{2} \cdot x\right)-1}{3}=2 \cdot F(x) \quad \text { mod. } 3,
$$

where:

$$
\begin{gathered}
F(x)=x+x^{3}+x^{9}+x^{27}+x^{81}+x^{243}+x^{729}+x^{2187}+x^{6561} \\
+x^{19683}+x^{59049}+O\left(x^{60000}\right)
\end{gathered}
$$

which is solution of

$$
x+F\left(x^{3}\right)=F(x) \quad \text { mod. } 3,
$$

i.e. $F(x)$ is an algebraic function modulo 3 :

$$
x+F(x)^{3}=F(x) \quad \bmod .3 .
$$

Unlike the situation on the ${ }_{3} F_{2}\left(\left[\frac{1}{9}, \frac{4}{9}, \frac{7}{9}\right],\left[\frac{1}{3}, 1\right], 27^{2} \cdot x\right)$ hypergeometric series, it is less obvious how to obtain the ${ }_{3} F_{2}\left(\left[\frac{1}{9}, \frac{4}{9}, \frac{5}{9}\right],\left[\frac{1}{3}, 1\right], 27^{2} \cdot x\right)$ as the diagonal of a rational function. It is however possible to obtain the solution of ${ }_{3} F_{2}\left(\left[\frac{1}{9}, \frac{4}{9}, \frac{5}{9}\right],\left[\frac{1}{3}, 1\right], 27^{2} \cdot x\right)$, as a telescoper of an algebraic function, and this solution is an algebraic function modulo $\mathrm{p}$.

4.2. A relation between ${ }_{3} F_{2}([1 / 9,4 / 9,5 / 9],[1 / 3,1], 27 \cdot x)$ and $a{ }_{4} F_{3}$ diagonal of an algebraic function

The diagonal of the product of algebraic functions

$$
\frac{(1-x-y)^{2 / 3}}{(1-x-y-z)} \cdot(1-w)^{-5 / 9}
$$

is given by the ${ }_{4} F_{3}$ hypergeometric function $\mathcal{H}$ which is the Hadamard product of ${ }_{3} F_{2}([1 / 9,4 / 9,7 / 9],[1 / 3,1], 27 \cdot x)$ and $(1-x)^{-5 / 9}$ :

$$
\begin{aligned}
\mathcal{H}={ }_{4} F_{3} & \left(\left[\frac{1}{9}, \frac{4}{9}, \frac{5}{9}, \frac{7}{9}\right],\left[\frac{1}{3}, 1,1\right], 27 \cdot x\right) \\
& =(1-x)^{-5 / 9} \star{ }_{3} F_{2}\left(\left[\frac{1}{9}, \frac{4}{9}, \frac{7}{9}\right],\left[\frac{1}{3}, 1\right], 27 \cdot x\right) \\
& =\operatorname{Diag}\left(\frac{(1-x-y)^{2 / 3}}{(1-x-y-z)} \cdot(1-w)^{-5 / 9}\right) .
\end{aligned}
$$

This ${ }_{4} F_{3}$ hypergeometric series (55) can also be written as the Hadamard product:

$$
\mathcal{H}=(1-x)^{-7 / 9} \star{ }_{3} F_{2}\left(\left[\frac{1}{9}, \frac{4}{9}, \frac{5}{9}\right],\left[\frac{1}{3}, 1\right], 27 \cdot x\right) .
$$


So even though we did not find a rational (or algebraic) function whose diagonal is given by (43), knowing that ${ }_{3} F_{2}\left(\left[\frac{1}{9}, \frac{4}{9}, \frac{7}{9}\right],\left[\frac{1}{3}, 1\right], 27 \cdot x\right)$ is the diagonal of a rational function, we see that the Hadamard product of (43) with a simple algebraic function $(1-x)^{-7 / 9}$ is actually a diagonal of an algebraic (or rational) function. This suggests but does not prove, that ${ }_{3} F_{2}\left(\left[\frac{1}{9}, \frac{4}{9}, \frac{5}{9}\right],\left[\frac{1}{3}, 1\right], 27 \cdot x\right)$ could also be a diagonal of a rational function.

\section{Conclusion}

Because of the crucial role played by diagonals of rational functions in physics [3, 4], Christol's conjecture is an important open problem. We have shown that the hypergeometric series ${ }_{3} F_{2}([2 / 9,5 / 9,8 / 9],[2 / 3,1], x)$ and ${ }_{3} F_{2}([1 / 9,4 / 9,7 / 9],[1 / 3,1], x)$ appearing in [4, 11] are diagonals of rational functions. We did so by first finding two algebraic functions whose diagonals were given by these two hypergeometric functions, and through an algorithm outlined in the paper [17, we were able to recover the rational functions whose diagonals are given by these two ${ }_{3} F_{2}$ hypergeometric functions.

We were also able to give a generalization of this result, and obtain other unresolved examples of Christol's conjecture as diagonals of rational functions. Furthermore, even though we were not able to write the ${ }_{3} F_{2}([1 / 9,4 / 9,5 / 9],[1 / 3,1], 27 \cdot x)$ given by Christol in [10], as a diagonal of a rational function, we gave two arguments that suggested that it was likely to be so. More generally, we believe after writing the ${ }_{3} F_{2}([2 / 9,5 / 9,8 / 9],[2 / 3,1], x)$ and ${ }_{3} F_{2}([1 / 9,4 / 9,7 / 9],[1 / 3,1], x)$ as diagonal of rational functions, that it is likely that the other ${ }_{3} F_{2}$ unresolved examples of Christol's conjecture are diagonals of rational functions.

Acknowledgments. J-M. M. and Y.A. would like to thank G. Christol for many enlightening discussions on diagonals of rational functions. We would like to thank A. Bostan for useful discussions on creative telescoping. Y.A. would like to thank A. Bostan for many explanations on Christol's conjecture including the details of 2.3 in an enlightening email correspondence [1. J-M. M. would like to thank the School of Mathematics and Statistics of Melbourne University where part of this paper was written for hospitality. J-M. M. would like to thank A.J. Guttmann for many discussions on D-finite series. We would like to thank A.J. Guttmann for proofreading the paper. Y.A. would like to thank J. Voight for providing enlightening explanations on Shimura curves and for providing reference [20]. C.K. was supported by the Austrian Science Fund (FWF): P29467-N32. Y. A. was supported by the Austrian Science Fund (FWF): F5011-N15. Y. A. would like to thank the RICAM for hosting him on several occasions. Y.A. would like to thank Elaine and Rob for their hospitality in Linz, and for the great discussions he had with them there. Y.A. would like to thank his parents and his family for all their support and love. We thank the Research Institute for Symbolic Computation for giving us access to the RISC software packages. 


\section{Appendix A. Counterexamples and links with Shimura curves}

The Gauss hypergeometric function appearing on the left in (9)

$$
{ }_{3} F_{2}\left(\left[\frac{2}{9}, \frac{5}{9}, \frac{8}{9}\right],\left[\frac{2}{3}, 1\right], 27 x\right)
$$

can be seen as the Hadamard product of a Gauss hypergeometric function and an algebraic function given by:

$$
{ }_{2} F_{1}\left(\left[\frac{2}{9}, \frac{5}{9}\right],\left[\frac{2}{3}\right], 27 x\right) \star(1-x)^{-8 / 9} .
$$

Now the Gauss hypergeometric function ${ }_{2} F_{1}\left(\left[\frac{2}{9}, \frac{5}{9}\right],\left[\frac{2}{3}\right], 27 x\right)$ happens to be a hypergeometric function corresponding to an automorphic form associated with a Shimura curve [24, 18, 19]. One has the identity:

$$
\begin{gathered}
{ }_{2} F_{1}\left(\left[\frac{2}{9}, \frac{5}{9}\right],\left[\frac{2}{3}\right], \quad 27 x\right)=(1-27 x)^{-1 / 9} \cdot\left(1-36 x+216 x^{2}\right)^{-1 / 18} \\
\times{ }_{2} F_{1}\left(\left[\frac{1}{36}, \frac{19}{36}\right],\left[\frac{8}{9}\right],-1728 \cdot \frac{x^{3} \cdot(1-27 x)}{\left(1-36 x+216 x^{2}\right)^{2}}\right) .
\end{gathered}
$$

The Gauss hypergeometric function ${ }_{2} F_{1}\left(\left[\frac{1}{36}, \frac{19}{36}\right],\left[\frac{8}{9}\right], x\right)$ can be also expressed as:

$$
{ }_{2} F_{1}\left(\left[\frac{1}{36}, \frac{19}{36}\right],\left[\frac{8}{9}\right], x\right)=(1-x)^{-1 / 36} \cdot{ }_{2} F_{1}\left(\left[\frac{1}{36}, \frac{13}{36}\right],\left[\frac{8}{9}\right],-\frac{x}{1-x}\right) .
$$

Now the Gauss hypergeometric function ${ }_{2} F_{1}\left(\left[\frac{1}{36}, \frac{13}{36}\right],\left[\frac{8}{9}\right], x\right)$ which occurs in p.14 of [21], corresponds to a hypergeometric function related to a Shimura curve since it has exponent differencest $(1 / 9,1 / 2,1 / 3)$, and these exponent differences are listed in the exhaustive list of hypergeometric functions that are associated with Shimura curves appearing in Table 1 of [20. Other ${ }_{3} F_{2}$ functions that are unresolved examples to Christol's conjecture that we found to be related to ${ }_{2} F_{1}$ hypergeometric functions related to Shimura curves are given by:

$$
\begin{aligned}
& { }_{3} F_{2}\left(\left[\frac{1}{9}, \frac{4}{9}, \frac{7}{9}\right],\left[\frac{4}{3}, 1\right], 3^{6} x\right)=(1-x)^{-1 / 9} \star{ }_{2} F_{1}\left(\left[\frac{4}{9}, \frac{7}{9}\right],\left[\frac{4}{3}\right], 3^{6} x\right), \\
& { }_{3} F_{2}\left(\left[\frac{2}{9}, \frac{5}{9}, \frac{7}{9}\right],\left[\frac{2}{3}, 1\right], 3^{6} x\right)=(1-x)^{-7 / 9} \star{ }_{2} F_{1}\left(\left[\frac{2}{9}, \frac{5}{9}\right],\left[\frac{2}{3}\right], 3^{6} x\right), \\
& { }_{3} F_{2}\left(\left[\frac{4}{9}, \frac{5}{9}, \frac{8}{9}\right],\left[\frac{2}{3}, 1\right], 3^{3} x\right)=(1-x)^{-8 / 9} \star{ }_{2} F_{1}\left(\left[\frac{4}{9}, \frac{5}{9}\right],\left[\frac{2}{3}\right], 3^{3} x\right), \\
& { }_{3} F_{2}\left(\left[\frac{1}{7}, \frac{2}{7}, \frac{4}{7}\right],\left[\frac{1}{2}, 1\right], 7^{4} x\right)=(1-x)^{-4 / 7} \star{ }_{2} F_{1}\left(\left[\frac{1}{7}, \frac{2}{7}\right],\left[\frac{1}{2}\right], 7^{4} x\right),
\end{aligned}
$$

Besides two hypergeometric functions, the ${ }_{3} F_{2}\left(\left[\frac{2}{9}, \frac{5}{9}, \frac{8}{9}\right],\left[\frac{2}{3}, 1\right], 27 x\right)$ and the ${ }_{3} F_{2}$ hypergeometric ${ }_{3} F_{2}\left(\left[\frac{1}{9}, \frac{4}{9}, \frac{7}{9}\right],\left[\frac{4}{3}, 1\right], 27 x\right)$, and the three globally bounded ${ }_{3} F_{2}$ hypergeometric series (A.6), (A.7) and (A.8), we were not able to write the other examples given in this section as a Hadamard product involving a ${ }_{2} F_{1}$ hypergeometric function associated to a Shimura curve. In any case, since the class of potential counterexamples formulated by Christol is infinite, while the list of Shimura in Table 1 of [20] is finite, a list of ${ }_{3} F_{2}$ functions both related to Shimura curves and to Christol's conjecture is bound to be finite.

$\dagger$ See 8] p.10 for a definition of exponent difference. 


\section{References}

[1] A. Bostan "Re: "Trivialement diagonale"". Message to Youssef Abdelaziz. 16 March 2019.

[2] A. Bostan, S. Boukraa, A.J. Guttmann, S. Hassani, I. Jensen, J-M. Maillard and Zenine High order Fuchsian equations for the square lattice Ising model: $\tilde{\chi}^{(5)}$ J. Phys. A 42 275209, (2009), http://arxiv.org/abs/0904.1601

[3] A. Bostan, S. Boukraa, G. Christol, S. Hassani, J-M. Maillard, Ising n-fold integrals as diagonals of rational functions and integrality of series expansions, (2013), J. Phys. A 46: Math. Theor. 185202 (44 pages), http://arxiv.org/abs/1211.6645v2

[4] A. Bostan, S. Boukraa, G. Christol, S. Hassani and J-M Maillard Ising $n$-fold integrals as diagonals of rational functions and integrality of series expansions: integrality versus modularity Preprint, (2012) http://arxiv.org/abs/1211.6031

[5] A. Bostan, S. Boukraa, S. Hassani, J-M. Maillard, J-A. Weil and N. Zenine Globally nilpotent differential operators and the square Ising model J. Phys. A 42 125206, (2009) http://arxiv.org/abs/0812.4931

[6] E. Goursat, Leccons sur les séries hypérgéométriques et sur quelques fonctions qui s'y rattachent, Hermann (Paris), (1938).

[7] F. Beukers, G. Heckman Monodromy for the hypergeometric function $n F n-1$ Inventiones mathematicae 95.2, 325-354 (2009)

[8] F-T. Tu, Algebraic Transformations of hypergeometric functions arising from theory of Shimura curves RIMS Kôkyûroku Bessatsu, B 44, pp.223-245 (2013).

[9] G. Christol, Diagonales de fractions rationnelles et équations de Picard-Fuchs, G.E.A.U. 12ème année, $1984 / 85, \mathrm{n}^{\circ} 13,12 \mathrm{p}$.

[10] G. Christol, Fonctions hypergéométriques bornées, (2013), Groupe de travail d'analyse ultramétrique, 14 (1986-1987), Exposé No. 8, 16 pp.

[11] G. Christol, Fonctions hypergéométriques et diagonales de fonctions rationnelles. La fonction ${ }_{3} F_{2}([1 / 9,4 / 9,5 / 9],[1 / 3,1], x)$ est-elle une diagonale? Conference "Journées holonomes", Institut Fourier, Grenoble, February 2014. Slides available at http://www-fourier.ujf-grenoble.fr/ jroques/journeesholonomes.html

[12] G. Christol Globally bounded solutions of differential equations Analytic number theory (Tokyo, 1988) (Lecture Notes in Math. vol 1434) (Berlin: Springer) pp. 45-64 http://dx.doi.org/10.1007/BFb0097124

[13] H.A. Schwarz Ueber diejenigen Fälle, in welchen die Gaussische hypergeometrische Reihe eine algebraische Function ihres vierten Elementes darstellt Journal für die reine und angewandte Mathematik (Crelle's Journal), Volume 1873 (75) Jan 1, 1873, 44 pages

[14] HolonomicFunctions Package version 1.7.1 (09-Oct-2013) written by Christoph Koutschan, Copyright 2007-2013, Research Institute for Symbolic Computation (RISC), Johannes Kepler University, Linz, Austria

[15] H. Furstenberg Algebraic functions over finite fields J. Algebra 7, 271-277 (1969) http://dx.doi.org/10.1016/0021-8693(67)90061-0

[16] HolonomicFunctions Package version 1.7.1 (09-Oct-2013) written by Christoph Koutschan, Copyright 2007-2013, Research Institute for Symbolic Computation (RISC), Johannes Kepler University, Linz, Austria

[17] J. Denef, L. Lipshitz, Algebraic power series and diagonals, J. Number Theory 26 46-67 http://dx.doi.org/10.1016/0022-314X(87)90095-3

[18] J. Voight, Shimura curves of genus at most two, Math. Comp. 78 pp 1155-1172, (2009).

[19] J. Voight, Three lectures on Shimura curves, 16th april (2006).

[20] K. Takeuchi, Commensurability classes of arithmetic triangle groups, 1977

[21] M. van Hoeij and R. Viduñas, Belyi functions for hyperbolic Hypergeometric-to-Heun transformations, Journal of Algebra, Volume 441, 1 November 2015, Pages 609-659, (2015) arXiv: 1212.3803v3

[22] S. Boukraa, S. Hassani, I. Jensen, J-M. Maillard and N. Zenine High order Fuchsian equations for the square lattice Ising model: $\tilde{\chi}^{(6)}$ J. Phys. A 43 115201, (2010), http://arxiv.org/abs/0912.4968

[23] T. Rivoal, J. Roques Hadamard products of algebraic functions, Journal of Number Theory, 145:579-603, (2014).

[24] Y. Abdelaziz, S. Boukraa, C. Koutschan, J-M. Maillard, Heun functions and diagonals of rational functions (unabridged version), arXiv:1910.10761v1

[25] Y. André G-functions and geometry Aspects of Mathematics E13 (Braunschweig: Friedr.Vieweg and Sohn) ISBN 3-528-06317-3 